\title{
A SURPRISING DYNAMICAL MASS FOR V773 Tau B
}

\author{
Andrew F. Boden ${ }^{1}$, Guillermo Torres ${ }^{2}$, Gaspard Duchêne ${ }^{3,4}$, Quinn Konopacky ${ }^{5,6}$, A. M. GheZ ${ }^{6}$, \\ Rosa M. TORRES S $^{7,8}$, AND LAURENT LOINARD 8,9 \\ ${ }^{1}$ Division of Physics, Mathematics, and Astronomy, California Institute of Technology, MS 11-17, Pasadena, CA 91125, USA \\ ${ }^{2}$ Harvard-Smithsonian Center for Astrophysics, 60 Garden Street, Cambridge, MA 02138, USA \\ ${ }^{3}$ Division of Astronomy and Astrophysics, University of California, Berkeley, CA 94720, USA \\ ${ }^{4}$ UJF-Grenoble 1/CNRS-INSU, Institut de Planétologie et d'Astrophysique de Grenoble (IPAG), UMR 5274, F-38041 Grenoble, France \\ ${ }^{5}$ Lawrence Livermore National Laboratory, 7000 East Avenue, Livermore, CA 94550, USA \\ ${ }^{6}$ Department of Physics and Astronomy, UCLA, Los Angeles, CA 90095-1562, USA \\ ${ }^{7}$ Argelander-Institut für Astronomie, Universität Bonn, Auf dem Hügel 71, D-53121 Bonn, Germany \\ ${ }^{8}$ Centro de Radiostronomía y Astrofísica, Universidad Nacional Autónoma de México, Apartado Postal 72-3 (Xangari), 58089 Morelia, Michoacán, México \\ ${ }^{9}$ Max-Planck-Institut für Radioastronomie, Auf dem Hügel 69, D-53121 Bonn, Germany \\ Received 2011 March 18; accepted 2011 November 29; published 2012 February 10
}

\begin{abstract}
We report on new high-resolution imaging and spectroscopy on the multiple T Tauri star system V773 Tau over the 2003-2009 period. With these data we derive relative astrometry, photometry between the A and B components, and radial velocity (RV) of the A-subsystem components. Combining these new data with previously published astrometry and RVs, we update the relative A-B orbit model. This updated orbit model, the known system distance, and A-subsystem parameters yield a dynamical mass for the B component for the first time. Remarkably, the derived $\mathrm{B}$ dynamical mass is in the range 1.7-3.0 $M_{\odot}$. This is much higher than previous estimates and suggests that like A, B is also a multiple stellar system. Among these data, spatially resolved spectroscopy provides new insight into the nature of the B component. Similar to A, these near-IR spectra indicate that the dominant source in B is of mid-K spectral type. If $B$ is in fact a multiple star system as suggested by the dynamical mass estimate, the simplest assumption is that B is composed of similar $\sim 1.2 M_{\odot}$ pre-main-sequence stars in a close ( $\left.<1 \mathrm{AU}\right)$ binary system. This inference is supported by line-shape changes in near-IR spectroscopy of B, tentatively interpreted as changing RV among components in V773 Tau B. Relative photometry indicates that B is highly variable in the near-IR. The most likely explanation for this variability is circum-B material resulting in variable line-of-sight extinction. The distribution of this material must be significantly affected by both the putative B multiplicity and the A-B orbit.
\end{abstract}

Key words: stars: fundamental parameters - stars: individual (V773 Tau) - stars: pre-main sequence

\section{INTRODUCTION}

V773 Tau (HDE 283447, HBC 367) is among the most remarkable pre-main-sequence (PMS) stellar systems presently known. V773 Tau exhibits a broad variety of observable properties traceable to its PMS status, circumstellar material, and multiplicity. V773 Tau was first identified as a T Tauri star by Rydgren et al. (1976) based on $\mathrm{H} \alpha$ and Ca II $H$ and $K$ emission, high lithium abundance, photometric variability, and $\mathrm{K} 2$ spectral type. The object presented an enigmatic mixture of classical and weak-lined T Tauri characteristics until it became clear there are multiple components; V773 Tau was resolved as a visual binary independently by Ghez et al. (1993) and Leinert et al. (1993) (with visual components designated here as A and B). Martín et al. (1994) first suggested and Welty (1995) established A as a short-period (51 days) double-lined spectroscopic binary (SB2). In filled-aperture high-angular resolution studies, Duchêne et al. (2003, hereafter D2003) and Woitas (2003) independently identified an additional, "infrared" component in the system (herein designated as C-note D2003 use an alternate component notation), making V773 Tau at least a compact quadruple system with no fewer than four stars within roughly $100 \mathrm{AU}$.

The SB2 A subsystem was resolved by near-IR (Boden et al. 2007, hereafter B2007) and radio (Phillips et al. 1996; B2007) interferometry, allowing physical orbit reconstruction and component dynamical mass estimates (B2007). Further, the variable radio emission and resolved morphology have been studied to infer magnetospheric interaction and complicated emission topology (Massi et al. 2002, 2006, 2008, and references therein). Finally in a companion paper to this one, the Very Long Baseline Array (VLBA) astrometric study of Torres et al. (2012, hereafter T2012) has refined the V773 Tau A orbit and revisited the system distance by both orbital and trigonometric parallax, indicating that the system is at $132.8 \pm 2.4 \mathrm{pc}$ (an earlier very long baseline interferometry trigonometric parallax by Lestrade et al. 1999 had estimated $148.4 \pm 5.5 \mathrm{pc}$, while the B2007 orbital analysis yielded $136.2 \pm 3.7 \mathrm{pc}$ ).

Since the D2003 results and modeling, near-IR imaging, and spectroscopic monitoring of V773 Tau (combined with B2007 results on $\mathrm{A}$ ) have yielded important additional clues to the nature of the B component. Here we will report on this continued monitoring, and what data taken over the past few years imply about the B component of this remarkable system.

\section{OBSERVATIONS}

We report on three types of new observations of V773 Tau. New resolved adaptive optics (AO)-corrected imaging from various telescopes provides relative astrometry and photometry among the V773 Tau components. New spectroscopic observations yielding radial velocity (RV) measurements of the two A constituents (following data presented in B2007) extend the time baseline for both A and A-B orbit modeling. Finally, AOresolved near-IR spectroscopy separates the emission from A and $\mathrm{B}$ for the first time, and appears to show photospheric lines from multiple stellar components for both A and B. We will discuss each data set in turn. 
Table 1

New V773 Tau Adaptive Optics Imaging Summary

\begin{tabular}{|c|c|c|c|c|c|c|c|c|c|}
\hline \multirow[t]{2}{*}{ Epoch } & \multirow[t]{2}{*}{ MJD } & \multirow[t]{2}{*}{ Telescope/Instrument } & \multirow[t]{2}{*}{ Filter } & \multicolumn{3}{|c|}{$\mathrm{A}-\mathrm{B}$} & \multicolumn{3}{|c|}{$\mathrm{A}-\mathrm{C}$} \\
\hline & & & & $\begin{array}{c}\rho \\
\text { (mas) }\end{array}$ & $\begin{array}{l}\text { P.A. } \\
\text { (deg) }\end{array}$ & $\begin{array}{c}\Delta m \\
(\mathrm{mag})\end{array}$ & $\begin{array}{c}\rho \\
\text { (mas) }\end{array}$ & $\begin{array}{l}\text { P.A. } \\
\text { (deg) }\end{array}$ & $\begin{array}{c}\Delta m \\
(\mathrm{mag})\end{array}$ \\
\hline 2004 Dec 19 & 53358 & KeckII/NIRC2 & $K$ & $112.8 \pm 1.9$ & $109.2 \pm 1.2$ & $1.44 \pm 0.15$ & $249 \pm 4$ & $151.7 \pm 1.4$ & $2.2 \pm 0.7$ \\
\hline 2006 Dec 24 & 54093 & VLT UT4/NACO & $K_{S}$ & $95.3 \pm 1.3$ & $121.9 \pm 1.3$ & $2.5 \pm 0.1$ & $254.5 \pm 1.3$ & $152.4 \pm 1.0$ & $2.08 \pm 0.02$ \\
\hline 2008 Oct 22 & 54761 & KeckII/NIRC2 & $K_{C}$ & $55.0 \pm 5.0$ & $137.9 \pm 5.4$ & $2.3 \pm 0.1$ & $250.0 \pm 1.8$ & $155.3 \pm 0.3$ & $1.95 \pm 0.03$ \\
\hline 2008 Oct 22 & 54761 & KeckII/NIRC2 & $K^{\prime}$ & $55.6 \pm 1.8$ & $143.7 \pm 1.4$ & $2.5 \pm 0.1$ & $249.1 \pm 1.0$ & $155.2 \pm 0.2$ & $2.10 \pm 0.02$ \\
\hline 2008 Dec 18 & 54818 & KeckII/NIRC2 & $\mathrm{Br}-\gamma$ & $49.0 \pm 3.0$ & $148.2 \pm 4.5$ & $2.7 \pm 0.1$ & $250.5 \pm 1.0$ & $155.6 \pm 0.3$ & $2.41 \pm 0.01$ \\
\hline 2009 Sep 9 & 55083 & KeckII/NIRC2 & $H_{C}$ & $48.0 \pm 2.0$ & $175.6 \pm 6.0$ & $3.3 \pm 0.2$ & $244.8 \pm 1.2$ & $156.3 \pm 0.3$ & $3.10 \pm 0.03$ \\
\hline 2009 Sep 9 & 55083 & KeckII/NIRC2 & $K^{\prime}$ & $45.0 \pm 2.0$ & $181.3 \pm 2.5$ & $3.0 \pm 0.2$ & $246.0 \pm 1.0$ & $156.9 \pm 0.2$ & $2.32 \pm 0.03$ \\
\hline 2009 Nov 29 & 55164 & KeckII/NIRC2 & $K^{\prime}$ & $49.0 \pm 4.0$ & $185.8 \pm 3.5$ & $3.1 \pm 0.2$ & $244.0 \pm 2.0$ & $156.5 \pm 0.3$ & $2.27 \pm 0.04$ \\
\hline
\end{tabular}

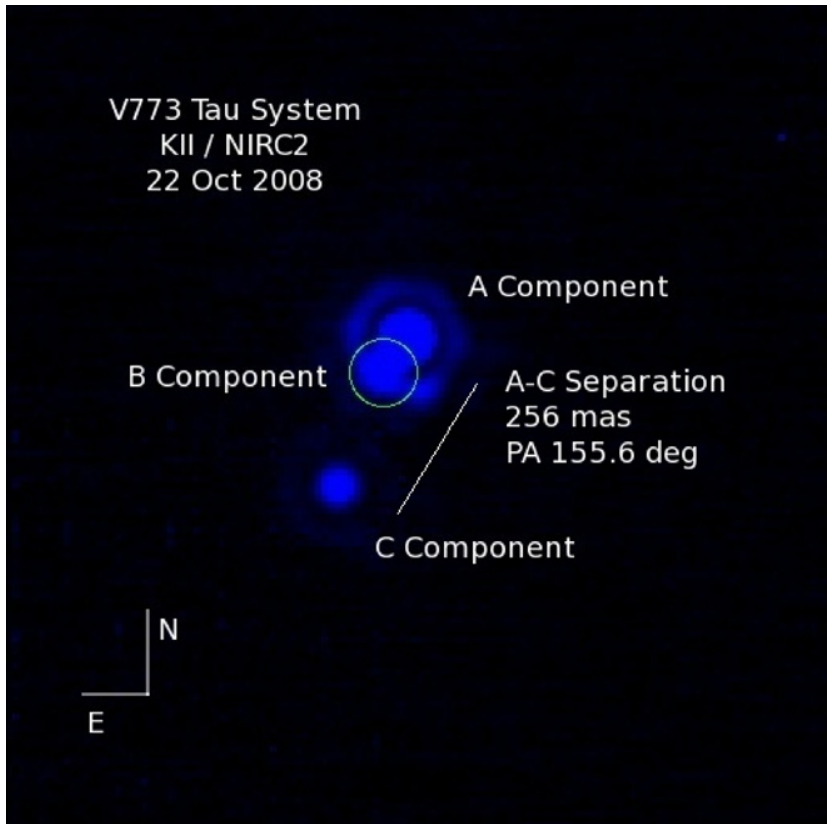

Figure 1. Keck II adaptive optics image of V773 Tau from 2008 October 22. The image is stretched to enhance low-level detail, so in addition to the A, $\mathrm{B}$, and $\mathrm{C}$ visual components, a hexagonal diffraction ring around the bright $\mathrm{A}$ component is apparent. At the epoch of this image the apparent A-B separation is roughly 50 mas at a position angle of $150^{\circ}$, and the apparent $\mathrm{A}-\mathrm{C}$ separation is roughly 260 mas at a position angle of $156^{\circ}$.

\subsection{Imaging}

Since the time of D2003 we have continued to monitor the V773 Tau system in imaging. As typical apparent spacings between the outer components in the system are well under an arcsecond, AO-corrected imaging is required to resolve these visual components. Figure 1 illustrates a spatially resolved $K$-band image of V773 Tau from 2008 October 22 obtained with the facility AO system (Wizinowich et al. 2000) and the NIRC2 instrument (PI: K. Matthews) on the Keck II telescope on Mauna Kea, HI.

Table 1 summarizes the eight new resolved imaging data sets available to us since D2003, including derived relative astrometry and relative photometry. Seven of these data sets were obtained at Keck with NIRC2, and one was produced by the Very Large Telescope/NACO system (Rousset et al. 2003; Lenzen et al. 2003). The relative astrometry and photometry among the $\mathrm{A}, \mathrm{B}$, and $\mathrm{C}$ components derived from these data were estimated by point-spread function (PSF) fitting with IRAF/DAOPHOT, except for the 2008-2009 epochs where the $\mathrm{A}$ and $\mathrm{B}$ components are within one diffraction ring radius (e.g., Figure 1). For these epochs, we used the inner $0.1^{\prime \prime}$ (the "core") of the C component as a PSF template to model A-component light and estimated B parameters based on residuals to this modeling. The plate scales for NIRC2 and NACO data were taken from Ghez et al. (2008) and Chauvin et al. (2010), respectively. Figure 2 depicts both older (D2003) and new (Table 1) relative A-B astrometry. Significant A-B orbital evolution since the D2003 summary is evident in the new astrometry; we discuss modeling the A-B orbit in Section 3.1.

Figure 3 depicts the relative photometry between the V773 Tau A and B components for data with centerband wavelengths within the near-IR $K$ band (2.0-2.4 $\mu \mathrm{m})$, including both older data from D2003 (D2003, Table 1) and the new imaging presented here. It is apparent that there is significant relative variability between the two components; the data indicate relative $K$ variability of up to $2.5 \mathrm{mag}$ - nearly a magnitude more than had been reported in D2003. D2003 argued that the A subsystem is photometrically stable, so presumably this large variability is due to the B component. D2003 further argued that this variability resulted from circumstellar material, an issue we will return to in Section 4.

\subsection{Radial Velocities}

Welty (1995) established V773 Tau A as an SB2 in spatially unresolved optical spectroscopy, and Welty (1995) and B2007 estimated the A orbit from RV measurements of both components. Remarkably, no signs of other V773 Tau components have been found in such optical spectroscopy-a point we will return to in Section 4. Since B2007 we have continued to monitor A in high-resolution optical spectroscopy and have obtained eight additional RV observations of both A components from the $1.5 \mathrm{~m}$ telescope on the Fred L. Whipple Observatory on Mt. Hopkins, AZ (see discussion in B2007). Further, the entire RV data set has been re-reduced for this analysis, now including both corrections for the systematic effects of finite spectrometer passband (a discussion of these corrections is given in Torres et al. 1997), and individual RV uncertainties estimated from the corresponding spectra signal-to-noise ratios (as opposed to ensemble statistics as was done in B2007). The resulting refinement of the A-subsystem orbit model with this revised RV set is discussed in T2012.

Additionally, for the purposes of A-B orbit analysis here the double-lined A RV measurements also serve to probe the A-barycenter kinematics, complementing the A-B astrometry. Straightforwardly, the A-barycenter RV is derivable from the A-component $\mathrm{RV}$ and the component mass ratio $\left(q_{\mathrm{A}} \equiv\right.$ 

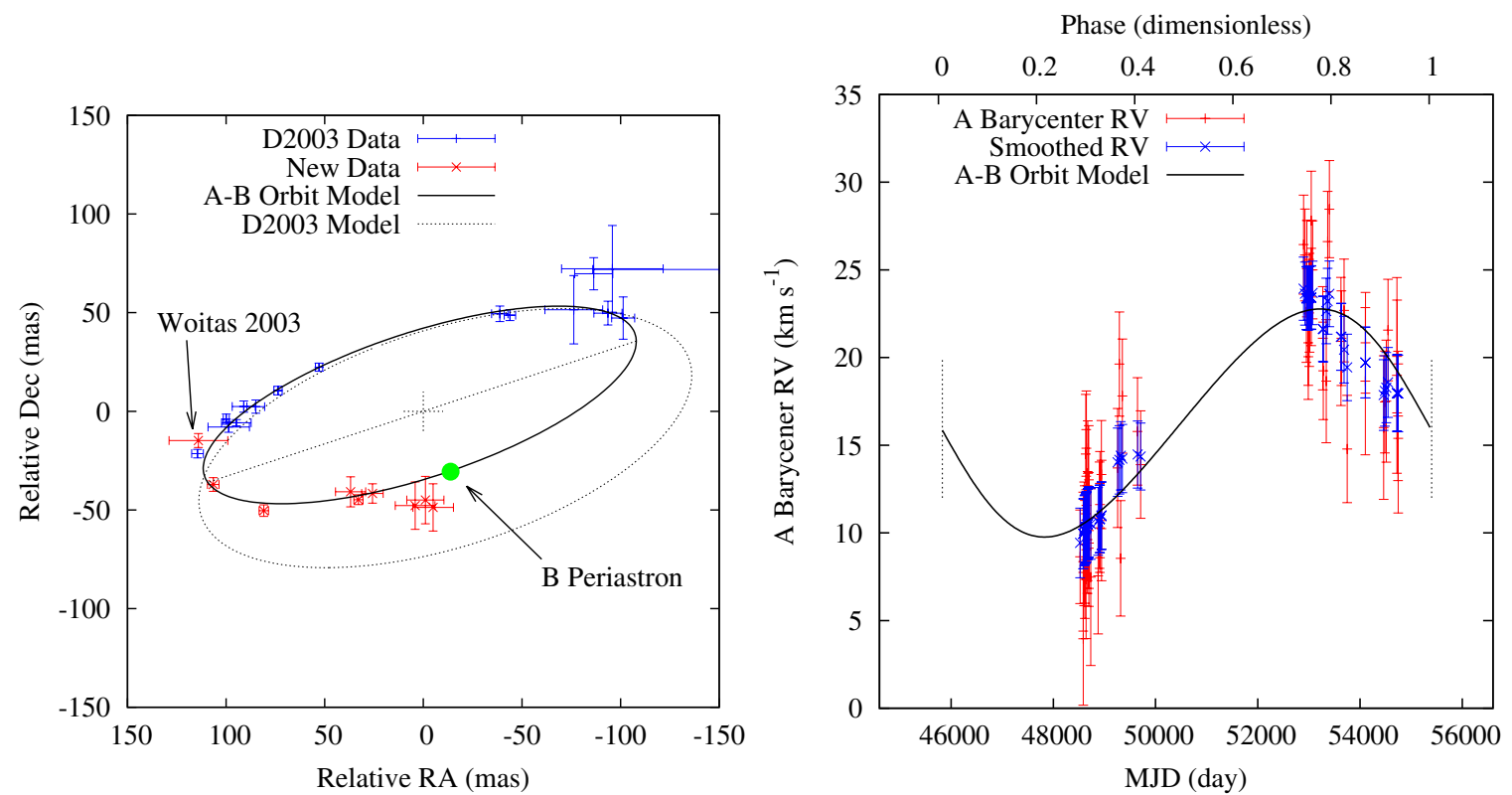

Figure 2. Astrometric and radial velocity data and orbit modeling for V773 Tau A-B. Left: we depict A-B relative astrometry (D2003 data in blue, new data in red-Table 1) and the best-fit A-B astrometric orbit models of D2003 and the present results (Table 3). Right: derived A-barycenter RV (Table 2) and our best-fit $\mathrm{RV}$ orbit model. Shown in red are the derived A-barycenter RV as used in the orbit modeling, and shown in blue are a version of these same data smoothed over the A-subsystem orbit period (51.1 days; B2007). The smoothed data are rendered here to illustrate how these data pertain to the longer-period A-B orbit; they are not used as input to the orbit modeling.

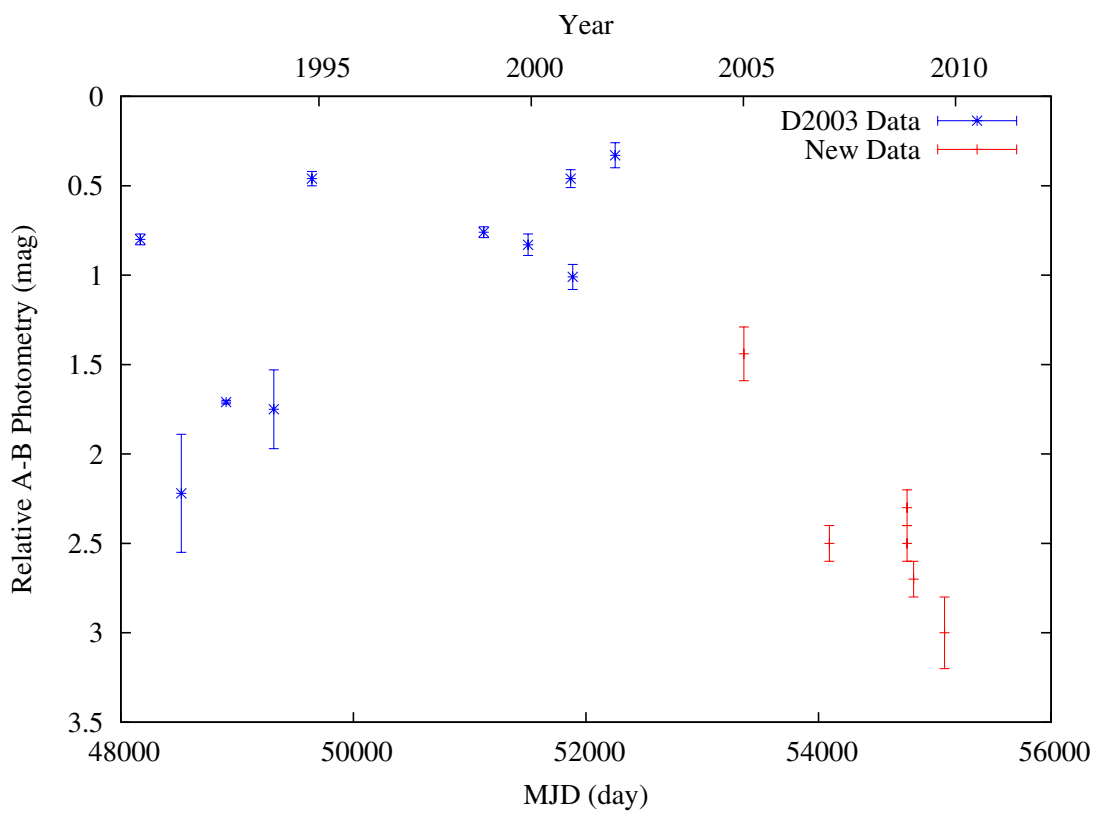

Figure 3. Relative V773 Tau A-B $K$-band photometry. Here we show relative $K$-band photometry between the A and B components derived from resolved imaging. Depicted in blue are older relative photometry data from D2003, Table 1; shown in red are new relative photometry data from this work (Table 1). Because the A component is thought to be photometrically stable (D2003), this variability is presumably in the B component.

$$
\begin{aligned}
& \left.M_{A b} / M_{A a}=K_{A a} / K_{A b}\right) \text { as } \\
& \mathrm{RV}_{A}=\frac{M_{A a} \mathrm{RV}_{A a}+M_{A b} \mathrm{RV}_{A b}}{M_{A a}+M_{A b}}=\frac{\mathrm{RV}_{A a}+q_{A} \mathrm{RV}_{A b}}{1+q_{A}} .
\end{aligned}
$$

Equation (1) codifies the coupling of the A- and A-B-subsystem orbits in the A-component RV observables. We return to the joint modeling of the A and A-B orbits with these observables in Section 3.1.

Table 2 gives a full listing of all A-subsystem component $\mathrm{RV}$ and derived A-barycenter RV for $q_{A}=0.831 \pm 0.031$ (see Section 3.1); these A-barycenter RV and A-B orbit models are depicted in Figure 2. (Note that the A-component velocities in Table 2 are presented without correction for A-subsystem motion. This is different from the presentation in B2007, where a model of the A-barycenter motion was removed from the velocities reported in B2007, Table 3.) The derived A-barycenter velocities show roughly $5 \mathrm{~km} \mathrm{~s}^{-1}$ of scatter on short timescales; this is consistent with the single-measurement precision of the V773 Tau Ab RV (see B2007, Table 3, and Table 2 here). But the derived A-barycenter velocities also show clear and variable acceleration over time, and a $13 \mathrm{~km} \mathrm{~s}^{-1}$ offset between the two $\mathrm{RV}$ data segments separated by $\sim 5000$ days. It is evident that 
Table 2

V773 Tau A RV Data Set

\begin{tabular}{|c|c|c|c|c|c|c|c|c|}
\hline MJD & $\mathrm{RV}_{A a}$ & $\mathrm{RV}_{A b}$ & $\sigma_{\mathrm{RV}-A a}$ & $\sigma_{\mathrm{RV}-A b}$ & $\mathrm{RV}_{A}$ & $\sigma_{\mathrm{RV}-A}$ & Model & Residuals \\
\hline 48524.4423 & -2.9 & 22.5 & 2.8 & 6.0 & 8.6 & 3.1 & 10.4 & -1.8 \\
\hline 48586.2579 & -16.9 & 29.1 & 4.0 & 8.5 & 4.0 & 4.5 & 10.5 & -6.6 \\
\hline 48587.2169 & -16.6 & 38.4 & 4.2 & 8.9 & 8.4 & 4.7 & 10.5 & -2.2 \\
\hline 48605.2134 & 22.3 & -6.2 & 3.7 & 7.9 & 9.4 & 4.2 & 10.6 & -1.2 \\
\hline 48613.3200 & 54.3 & -44.7 & 3.5 & 7.4 & 9.3 & 3.9 & 10.6 & -1.2 \\
\hline 48616.1975 & 49.5 & -40.9 & 3.5 & 7.4 & 8.5 & 4.0 & 10.6 & -2.0 \\
\hline 48635.1851 & -14.2 & 49.0 & 3.6 & 7.6 & 14.5 & 4.0 & 10.6 & 3.9 \\
\hline 48640.1978 & -20.8 & 41.2 & 3.6 & 7.5 & 7.3 & 4.0 & 10.6 & -3.2 \\
\hline 48644.0806 & -8.7 & 33.1 & 3.1 & 6.6 & 10.3 & 3.5 & 10.6 & -0.3 \\
\hline 48644.1505 & -11.1 & 40.8 & 3.6 & 7.7 & 12.5 & 4.0 & 10.6 & 1.8 \\
\hline 48647.1902 & -6.7 & 40.8 & 3.4 & 7.3 & 14.8 & 3.8 & 10.6 & 4.2 \\
\hline 48667.1397 & 49.0 & -37.8 & 3.2 & 6.8 & 9.6 & 3.6 & 10.7 & -1.1 \\
\hline 48670.0901 & 34.9 & -19.1 & 3.3 & 6.9 & 10.4 & 3.7 & 10.7 & -0.3 \\
\hline 48675.1315 & 0.4 & 29.0 & 3.2 & 6.7 & 13.4 & 3.5 & 10.7 & 2.7 \\
\hline 48693.1130 & -15.5 & 46.4 & 3.7 & 7.8 & 12.6 & 4.1 & 10.7 & 1.9 \\
\hline 48695.1549 & -13.7 & 37.2 & 3.8 & 8.1 & 9.4 & 4.2 & 10.7 & -1.3 \\
\hline 48697.1190 & -8.0 & 32.0 & 3.5 & 7.4 & 10.1 & 3.9 & 10.7 & -0.6 \\
\hline 48732.1320 & -15.1 & 34.6 & 5.3 & 11.3 & 7.5 & 5.9 & 10.8 & -3.3 \\
\hline 48875.4835 & 29.3 & -18.7 & 3.5 & 7.4 & 7.6 & 3.9 & 11.1 & -3.6 \\
\hline 48902.4818 & -8.6 & 34.6 & 3.2 & 6.8 & 11.0 & 3.6 & 11.2 & -0.2 \\
\hline 48910.3196 & 18.0 & 1.0 & 2.7 & 5.7 & 10.3 & 3.0 & 11.2 & -0.9 \\
\hline 48913.4884 & 27.6 & -7.6 & 3.2 & 6.8 & 11.6 & 3.6 & 11.2 & 0.4 \\
\hline 48939.4015 & -10.5 & 41.9 & 3.2 & 6.9 & 13.3 & 3.6 & 11.3 & 2.0 \\
\hline 48944.3441 & -17.1 & 44.1 & 3.6 & 7.7 & 10.7 & 4.0 & 11.3 & -0.6 \\
\hline 49259.5194 & -9.7 & 41.8 & 3.6 & 7.6 & 13.7 & 4.0 & 12.2 & 1.5 \\
\hline 49291.3832 & 4.3 & 38.1 & 3.1 & 6.7 & 19.6 & 3.5 & 12.2 & 7.4 \\
\hline 49318.2384 & 15.5 & 0.1 & 3.5 & 7.4 & 8.5 & 3.9 & 12.3 & -3.7 \\
\hline 49351.2823 & -11.0 & 52.4 & 3.4 & 7.3 & 17.8 & 3.8 & 12.4 & 5.4 \\
\hline 49648.4224 & 2.1 & 32.3 & 3.2 & 6.9 & 15.8 & 3.6 & 13.4 & 2.4 \\
\hline 49707.1592 & -10.8 & 43.7 & 3.2 & 6.9 & 13.9 & 3.6 & 13.5 & 0.4 \\
\hline 52894.4751 & 17.2 & 37.6 & 3.0 & 6.4 & 26.4 & 3.3 & 22.6 & 3.8 \\
\hline 52923.4044 & 6.4 & 49.3 & 2.8 & 5.9 & 25.8 & 3.1 & 22.7 & 3.2 \\
\hline 52951.4236 & 48.2 & -8.2 & 3.0 & 6.4 & 22.6 & 3.4 & 22.7 & -0.1 \\
\hline 52959.3510 & 64.2 & -22.0 & 2.8 & 6.0 & 25.1 & 3.2 & 22.7 & 2.4 \\
\hline 52986.3147 & -4.3 & 51.0 & 2.5 & 5.4 & 20.8 & 2.9 & 22.7 & -1.9 \\
\hline 52987.3316 & -0.3 & 44.6 & 2.6 & 5.4 & 20.1 & 2.9 & 22.7 & -2.6 \\
\hline 53007.2212 & 66.9 & -30.8 & 3.1 & 6.7 & 22.5 & 3.6 & 22.7 & -0.2 \\
\hline 53013.2445 & 49.2 & -8.3 & 3.0 & 6.3 & 23.1 & 3.3 & 22.7 & 0.4 \\
\hline 53044.1602 & 9.6 & 49.7 & 3.0 & 6.3 & 27.8 & 3.3 & 22.7 & 5.1 \\
\hline 53046.1895 & 11.8 & 37.5 & 2.9 & 6.2 & 23.5 & 3.3 & 22.7 & 0.7 \\
\hline 53073.1090 & 9.7 & 43.4 & 3.0 & 6.3 & 25.0 & 3.3 & 22.7 & 2.3 \\
\hline 53272.5076 & 32.0 & 8.0 & 3.1 & 6.6 & 21.1 & 3.5 & 22.8 & -1.7 \\
\hline 53282.4624 & -1.9 & 44.7 & 2.9 & 6.2 & 19.2 & 3.3 & 22.8 & -3.5 \\
\hline 53339.3984 & -3.4 & 45.1 & 3.7 & 7.9 & 18.7 & 4.1 & 22.8 & -4.1 \\
\hline 53365.3371 & 69.1 & -24.5 & 2.9 & 6.2 & 26.6 & 3.4 & 22.7 & 3.8 \\
\hline 53399.1436 & 4.1 & 57.7 & 2.9 & 6.2 & 28.5 & 3.3 & 22.7 & 5.7 \\
\hline 53626.4715 & 49.4 & -13.3 & 3.8 & 8.2 & 20.9 & 4.3 & 22.5 & -1.6 \\
\hline 53634.4855 & 9.7 & 34.9 & 2.9 & 6.1 & 21.1 & 3.2 & 22.5 & -1.4 \\
\hline 53686.4318 & 7.8 & 40.7 & 3.1 & 6.6 & 22.7 & 3.4 & 22.4 & 0.3 \\
\hline 53748.3187 & -7.9 & 42.1 & 3.2 & 6.9 & 14.8 & 3.6 & 22.3 & -7.6 \\
\hline 54104.2620 & -4.4 & 46.4 & 4.4 & 9.4 & 18.7 & 4.9 & 21.5 & -2.9 \\
\hline 54107.2656 & -3.4 & 50.1 & 3.0 & 6.4 & 20.8 & 3.4 & 21.5 & -0.7 \\
\hline 54461.3453 & -6.3 & 42.9 & 4.3 & 9.2 & 16.0 & 4.8 & 20.4 & -4.3 \\
\hline 54485.2461 & 45.6 & -16.3 & 3.0 & 6.5 & 17.5 & 3.4 & 20.3 & -2.7 \\
\hline 54514.2607 & -5.2 & 44.8 & 3.7 & 7.8 & 17.5 & 4.1 & 20.1 & -2.6 \\
\hline 54546.1377 & 47.2 & -9.3 & 3.0 & 6.5 & 21.6 & 3.4 & 20.0 & 1.6 \\
\hline 54721.4846 & -5.1 & 48.0 & 5.9 & 12.6 & 19.0 & 6.6 & 19.3 & -0.3 \\
\hline 54723.4654 & -5.3 & 50.5 & 3.4 & 7.2 & 20.1 & 3.8 & 19.2 & 0.8 \\
\hline 54743.4370 & 57.5 & -32.4 & 3.8 & 8.2 & 16.7 & 4.3 & 19.2 & -2.5 \\
\hline 54746.4858 & 59.6 & -37.7 & 4.4 & 9.4 & 15.4 & 5.0 & 19.1 & -3.8 \\
\hline
\end{tabular}

Notes. Units of all RV in $\mathrm{km} \mathrm{s}^{-1}$. Residuals are quoted as data minus model. A-barycenter RVs are computed assuming $q_{A}=0.831$ (Section 3.1). Note that unlike B2007 the A-component velocities are presented without correction for A-subsystem motion. 
Table 3

Orbital Parameters for V773 Tau A-B

\begin{tabular}{lccc}
\hline \hline $\begin{array}{l}\text { Orbital } \\
\text { Parameter }\end{array}$ & T2002 & D2003 & $\begin{array}{c}\text { Joint Solution } \\
\text { (This Work) }\end{array}$ \\
\hline Period (yr) & $125.0 \pm 6.0$ & $46.0 \pm 6.0$ & $26.20 \pm 1.1$ \\
$T_{0}(\mathrm{yr})$ & $1998.62 \pm 3.0$ & $1996.5 \pm 0.8$ & $2010.53 \pm 1.0$ \\
$e$ & $0.643 \pm 0.04$ & $0.30 \pm 0.10$ & $0.099 \pm 0.026$ \\
$K_{A}\left(\mathrm{~km} \mathrm{~s}^{-1}\right)$ & & & $6.50 \pm 0.50$ \\
$\gamma\left(\mathrm{km} \mathrm{s}^{-1}\right)$ & & & $16.30 \pm 0.51$ \\
$\omega_{A}(\mathrm{deg})$ & $299.4 \pm 10.0$ & $288 \pm 1$ & $94 \pm 17$ \\
$\Omega(\mathrm{deg})$ & $101.1 \pm 7$ & $66 \pm 3$ & $288.2 \pm 1.0$ \\
$i(\mathrm{deg})$ & $63.8 \pm 5$ & $140 \pm 10$ & $71.48 \pm 0.78$ \\
$a(\mathrm{mas})$ & $249 \pm 15$ & & $115.5 \pm 3.4$
\end{tabular}

Notes. Summarized here are the orbital parameters for the A-B subsystem as estimated by T2002, D2003, and present results from our joint modeling of the V773 Tau A and A-B orbits. $\omega_{A}$ is the argument of periastron for the A subsystem, and $\Omega$ is quoted in a position-angle convention. $\Omega$ and $\omega_{A}$ from T2002 appear particularly discrepant from the other work summarized here. We present these parameters as they are listed in T2002, Table 2, but the general agreement of the A-B orbit orientation depicted in their Figure 1 and our Figure 2 leads us to speculate that $\Omega$ and $\omega_{A}$ may have been reversed as they are listed in T2002_-bringing them into much better agreement with other results.

these derived A-barycenter velocities contain useful kinematic information for modeling the A-B orbit.

\subsection{Spatially Resolved Near-infrared Spectroscopy}

In order to determine the spectral type of V773 Tau B, and in an attempt to resolve the A component into a near-infrared double-lined spectroscopic binary for the first time, we observed V773 Tau using the near-infrared cross-dispersed spectrograph NIRSPEC (McLean et al. 2000) behind the Keck II AO system in two epochs (2003 December 11 and 2006 December 16 , Table 1$)$. In both cases, we obtained $K$-band spectra aligning the slit with the A-B subsystem. We obtained six $150 \mathrm{~s}$ integrations with the 0.013 slit in 2003 and four $300 \mathrm{~s}$ frames with the 0 '.041 slit in 2006 in A-B dither patterns along the length of the slit. In both epochs, we observed an early A-type star (HD 27962/HR 1389 in 2003, HD 34203/HR 1718 in 2006) immediately following V773 Tau to estimate the telluric transmission. The resolution of the spectra from 2003 is $R \sim 40,000$ and from 2006 is $R \sim 25,000$.

The basic data reduction was performed with REDSPEC, a software package designed for NIRSPEC. ${ }^{10}$ Object frames are reduced by subtracting opposing nods to remove sky and dark backgrounds, dividing by a flat field, and correcting for bad pixels. Individual spectral orders are spatially rectified by fitting the trace of the A0 calibrators with third-order polynomials which are then applied to target images. The wavelength solution is determined using the etalon lamps that are part of the NIRSPEC lamp suite (Figer et al. 2003). The absolute values of the etalon lines are calibrated using the telluric features in our A0 calibrators (Konopacky et al. 2010). The wavelength solution is modeled with a second-order polynomial.

Extraction of the A and B components from the reduced and rectified frames is a challenge as cross-contamination is possible. This is particularly true of the 2006 epoch, where the separation was less than 0 !' 1 and the A/B flux ratio had increased from previous epochs (e.g., Table 1 and Figure 3). In both 2003 and 2006, we opted to extract the spectra by fitting a Gaussian to

\footnotetext{
$\overline{10}$ http://www2.keck.hawaii.edu/inst/nirspec/redspec/index.html
}

the trace of one visual component and subtracting the fit result from the frame to leave only the other component. In 2003, we allowed the FWHM of the Gaussian to vary with wavelength. In 2006, the two components are too closely blended to fit for the FWHM; instead, we fixed the FWHM to the value found for the telluric calibrator. The remaining trace in the frame was then extracted by multiplying the flux by a normalized Gaussian fit by the same method (Konopacky et al. 2010). We then removed the telluric features from the spectra by dividing by the extracted A0 calibrator spectra. In order to use the A0 star to correct the order containing $\mathrm{Br} \gamma$, we fit the $\mathrm{Br} \gamma$ line in the $\mathrm{A} 0$ stars with a Lorentzian profile, which we subtracted out of the spectrum before dividing.

There are four useful orders for which spectra were extracted, covering the approximate ranges $2.10-2.13 \mu \mathrm{m}, 2.16-2.19 \mu \mathrm{m}$, $2.22-2.26 \mu \mathrm{m}$, and $2.29-2.32 \mu \mathrm{m}$. Samples of these spectral orders are given in Figure 4.

\section{ANALYSIS}

\subsection{A-B Orbit Modeling}

At the time of D2003 considerable orbital evolution was apparent in resolved imaging (and resulting relative astrometry) of the V773 Tau A-B pair (e.g., Tamazian et al. 2002; Woitas 2003; D2003; Figure 2), but it had not yet completed even half a full orbit since the 1990 discovery of B (Ghez et al. 1993). Both Tamazian et al. (2002, hereafter T2002) and D2003 made preliminary estimates of the A-B orbit based on early astrometry, but with the limited phase coverage of the data these two analyses came to significantly different conclusions about the A-B orbit.

In the present work, we have augmented the D2003 astrometry data set with the new astrometry presented above (Table 1, plus one new astrometric point presented in Woitas 2003) and the RV observations presented above (Table 2). Figure 2 depicts expanded relative astrometric and RV data sets and our updated orbital modeling (Table 3) for V773 Tau A-B. In particular, the left panel depicts the available set of relative astrometry on the A-B pair, and both the D2003 and our orbit model visual trace. Older astrometry summarized in D2003 (specifically D2003, Table 1) is rendered in blue, while newer astrometry derived from imaging reported here is rendered in red. Figure 2 (right panel) shows (derived) A-subsystem barycentric RV (Section 2.2; Table 2) and the RV trace from our model orbit. Figure 2 (right) also shows a rendering of the A-barycenter RV smoothed at the A-subsystem period (51.1 days; B2007). This rendering is provided to illustrate the A-barycenter RV content relevant to the $\mathrm{A}-\mathrm{B}$ orbit modeling; these smoothed data are not used in the orbit modeling.

Table 3 lists the parameters for the A-B orbit as estimated here and in the earlier work from T2002 and D2003. Using input relative astrometry (e.g., Table 1) and A-barycenter RV (Table 2) we made initial estimates of the A-B orbit using both Marquardt-Levenberg least-squares and Bayesian modeling techniques, and found good agreement in the results from these two methods (see orbit modeling descriptions in Boden et al. 2000, Torres et al. 2002, and Boden et al. 2005). Our final refinement of the A-B orbit model was made by joint modeling of both the A- and A-B-subsystem orbits simultaneously, coupling the A-component RV data to the two subsystem orbits through Equation (1), and adding A-subsystem relative astrometry data from the Keck Interferometer (B2007) and VLBA (B2007; T2012) sources. In practice 

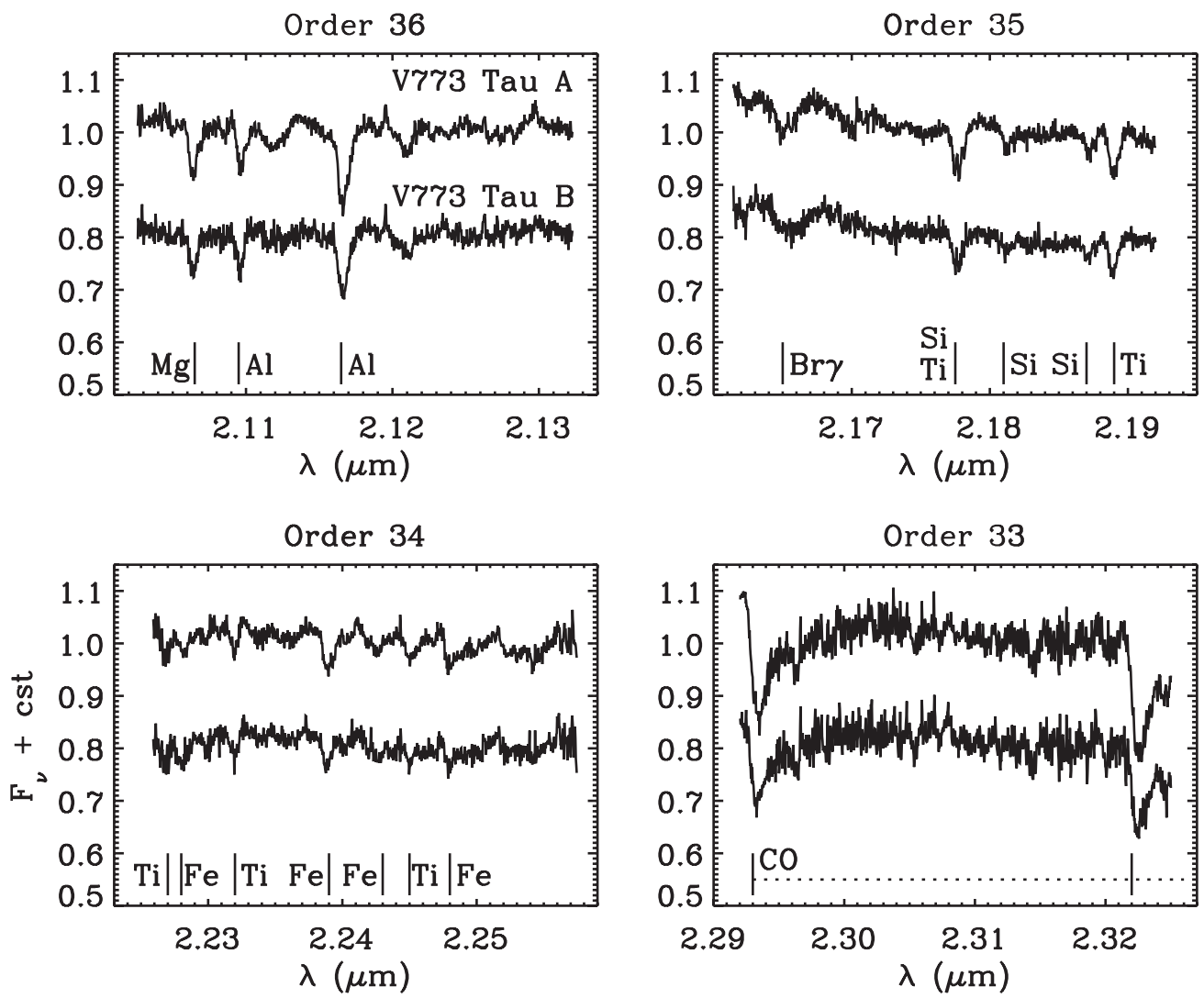

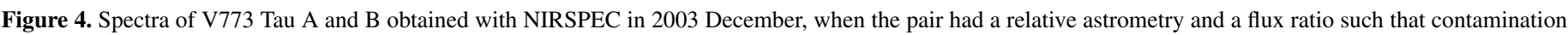

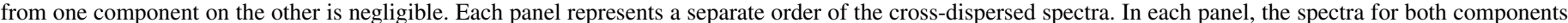

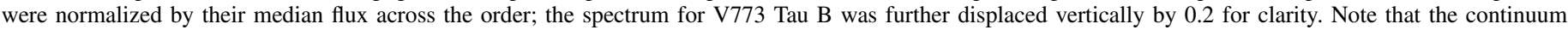

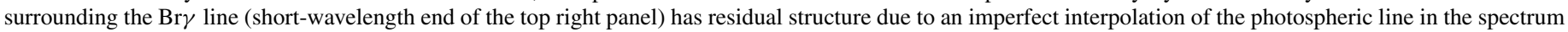
of the telluric standard.

the joint modeling was accomplished by iterative application of Marquardt-Levenberg optimization for the A and A-B models in turn, coordinating the $\mathrm{A}$ and $\mathrm{A}-\mathrm{B} \mathrm{RV}$ observables through Equation (1) between iterations. This process converged in only a few (four) iterations and resulted in stable solutions for both orbits. The A-subsystem orbit solution is described in the companion paper (T2012) and is found to be in good statistical agreement with the earlier estimate from B2007. It is both notable and relevant to the $\mathrm{A}-\mathrm{B}$ results that the new $\mathrm{RV}$ reduction and joint $\mathrm{A} / \mathrm{A}-\mathrm{B}$ modeling yield a subtly different value for $q_{A}(0.831 \pm 0.031)$ than found in B2007 $(0.865 \pm 0.032)$. Additional details on the final A orbit model can be found in T2012.

Focusing here on the A-B portion of the joint solution, we find significant differences between our A-B orbit modeling results and those published previously by T2002 and D2003, e.g., see the visual orbits rendered in Figure 2. Prima facie these differences are due to the greatly expanded data set available here to estimate the A-B orbit. Our modeling is supported both by expanded phase coverage in the relative astrometry (e.g., Figure 2) and the addition of complementary A-barycenter RV data, neither of which was available in earlier work. At the present epoch the astrometric data set covers roughly $73 \%$ of the estimated orbit period, compared to only $46 \%$ for data available to D2003. Further, the new astrometric data are seen to be particularly important in revealing the orbit's character: Figure 2 shows that the A-B apparent orbit passed maximum elongation around the time of D2003, and since that time has exhibited remarkably rapid position-angle evolution as the system approaches periastron at roughly the present epoch
(Table 3). These differences are most pronounced in the A-B orbit period-our present period estimate (26.2 yr) is roughly half of the value estimated by D2003 and a factor of five smaller than estimated by T2002. Finally, the addition of the A-subsystem RV is seen to strongly support the updated A-B orbit modeling.

\subsection{V773 Tau B Dynamical Mass}

Typically, a relative astrometric orbit such as derived here requires the support of radial velocities for both stars (e.g., Torres et al. 2002; B2007, and references therein) in order to yield unambiguous component dynamical masses. However V773 Tau A-B is unique in that it is supported by the Asubsystem binarity, and the analyses of the A subsystem by B2007 and T2012 - providing both A-subsystem total mass and distance. When combined with these two items the orbit model in Table 3 allows an unambiguous estimate for the B-component dynamical mass.

Table 4 summarizes the computed physical properties for the A-B components. In particular the new quantities provided by the A-B orbit are the A-B physical semimajor axis and $\mathrm{B}$ dynamical mass. Adopting the T2012-favored composite system distance of $132.8 \pm 2.4 \mathrm{pc}$, the resulting A-B semimajor axis is $15.35 \pm 0.45 \mathrm{AU}$, and the $\mathrm{B}$ dynamical mass is $2.35 \pm 0.67 M_{\odot}$. This large value for the B-component mass is completely unexpected (e.g., see discussion in D2003) and makes B's mass comparable to that for A. However, note that the T2012-estimated A-subsystem mass corresponds to an A-subsystem orbital distance of $135.7 \pm 3.2 \mathrm{pc}$. Using the 
Table 4

Physical Parameters for V773 Tau A-B

\begin{tabular}{lcc}
\hline \hline Parameter & Value & Note \\
\hline System distance $(\mathrm{pc})$ & $132.8 \pm 2.4$ & T2012 \\
A-subsystem mass $\left(M_{\odot}\right)$ & $2.91 \pm 0.20$ & B2007; T2012 \\
A-subsystem luminosity $\left(L_{\odot}\right)$ & $3.93 \pm 0.38$ & B2007 \\
A-B semimajor axis $(\mathrm{AU})$ & $15.35 \pm 0.45$ & \\
A-B system mass $\left(M_{\odot}\right)$ & $5.27 \pm 0.65$ & \\
B mass $\left(M_{\odot}\right)$ & $2.35 \pm 0.67$ & Section 3.2 \\
B luminosity $\left(L_{\odot}\right)$ & $2.6 \pm 0.6$ & Section 4 \\
\hline
\end{tabular}

Notes. Summarized here are the physical parameters for the A-B subsystem as given by previous results and derived here. Note we use the preferred composite distance estimate from T2012 as the basis for these computations, but other variations are possible - please refer to comments in the text for more detail.

A-subsystem orbital distance to interpret the A-B orbit presented here (thereby placing the A and B dynamical masses on a consistent distance basis), the resulting semimajor axis and $\mathrm{B}$ mass are $15.67 \pm 0.46 \mathrm{AU}$ and $2.69 \pm 0.67$, respectively. While we recommend the former values based on the T2012 composite distance estimate as probably more accurate, the latter values represent viable alternate (and statistically consistent) estimates based on present data.

The companion VLBA study of T2012 offers the unique opportunity to cross-check our B dynamical mass estimate through independent means. In modeling the VLBA data on V773 Tau A, T2012 find it necessary to account for the A-B orbital motion as an effective mean acceleration over the time interval of their data. This situation is depicted in Figure 5. At each of the epochs of the T2012 VLBA observations the B mass accelerates A toward B's position. With our A-B orbit model (Table 3), B dynamical mass, and system distance (Table 4) we can estimate the instantaneous A acceleration, and the resulting average acceleration over the 27 VLBA epochs (T2012, Table 1). When we do, we estimate the average skyprojected acceleration to be $a_{\alpha} \cos \delta=2.28 \pm 0.65$ mas $\mathrm{yr}^{-2}$ and $a_{\delta}=-1.27 \pm 0.36$ mas $\mathrm{yr}^{-2}$ As shown in Figure 5, this value is well within $1 \sigma$ agreement with the VLBA-measured

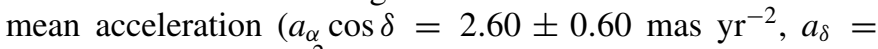
$-1.51 \pm 0.52$ mas $\mathrm{yr}^{-2}$; vector and uncertainty ellipse rendered in blue) from T2012. The consistency of these two independent acceleration estimates implies that our A-B orbit model and B dynamical mass are reliable at their stated uncertainties.

\subsection{Near-infrared Spectroscopic Results for V773 Tau B}

The extracted NIRSPEC spectra for V773 Tau A and B are shown in Figure 4. At first glance, the $K$-band spectra of V773 Tau A and B appear very similar, suggesting that the B component has a K-type spectral type, similar to A. Unfortunately, our cross-dispersed spectra do not include the strongest photospheric features of late-type stars ( $\mathrm{Na}$ doublet at $2.20 \mu \mathrm{m}$ and $\mathrm{Ca}$ triplet at $2.26 \mu \mathrm{m}$ ), so we have to rely on somewhat weaker features to estimate the spectral type of V773 Tau B. The strongest features in our spectra are an Mg doublet and two $\mathrm{Al}$ lines in order $36, \mathrm{H} \mathrm{Br} \gamma, \mathrm{Si}, \mathrm{Ti}$, and $\mathrm{Fe}$ lines in order 35 , weak Fe and $\mathrm{Ti}$ lines in order 34 , and the $\mathrm{CO}(2-0)$ forest and the first two band heads in order 33 . The various line ratios are very similar for components $\mathrm{A}$ and $\mathrm{B}$, although all lines appear weaker in B than in A. This may be due to contamination by emission from hot circumstellar dust (veiling) or by the presence of a hotter (hence almost featureless) unresolved component.

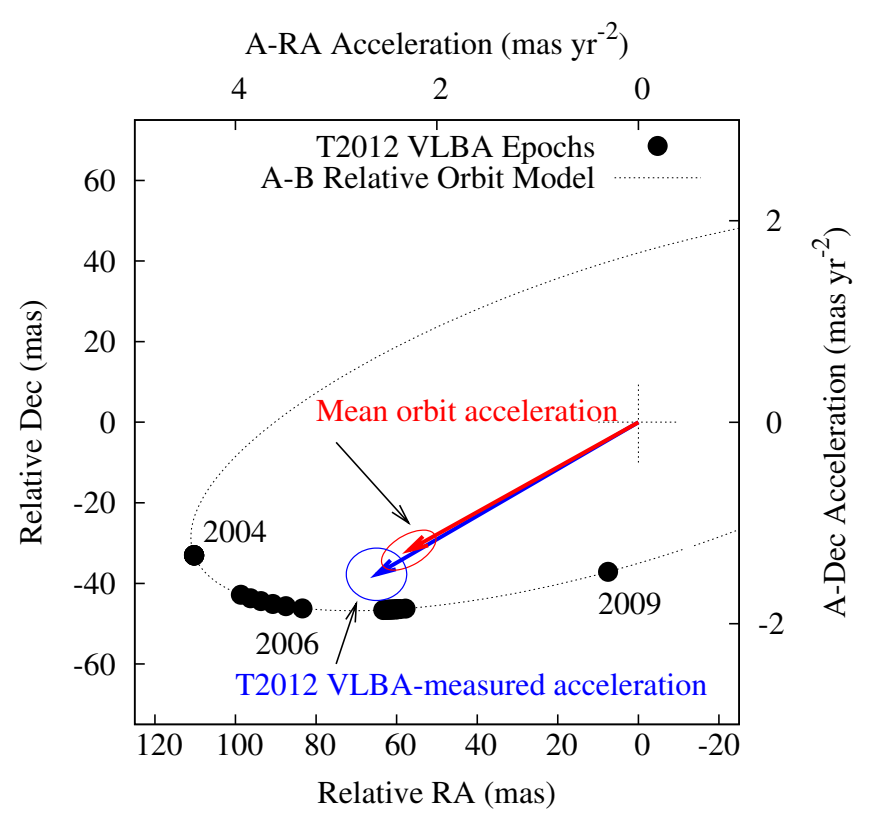

Figure 5. V773 Tau A acceleration estimates. The orbital acceleration on A produced by B is measured by the VLBA astrometry reported by T2012. The relative A-B position is shown here for the 27 T2012 VLBA measurement epochs; we can use our A-B physical orbit model and the component (A: B2007; T2012, and B: this work) dynamical mass values to estimate the mean acceleration in the T2012 data. The mean A acceleration estimates from T2012 (blue) and our orbit model (red) are shown along with their $1 \sigma$ uncertainty ellipses. The two acceleration estimates are seen to be in excellent (i.e., well within $1 \sigma$ ) agreement, providing an independent confirmation of our orbit model and $\mathrm{B}$ dynamical mass estimate.

We compared our spectra to several published libraries of $K$ band spectra for field stars (Kleinman \& Hall 1986; Wallace \& Hinkle 1996; Ivanov et al. 2004; Rayner et al. 2009) as well as to the Gemini/GNIRS spectral library ${ }^{11}$ to estimate the spectral type of both components. For the A component we infer a K1-K3 spectral type in good agreement with the system's consensus spectral typing, while for the B component we find a K2-K5 spectral type. The slightly later type for B is driven by (1) the slightly weaker Si line at $2.187 \mu \mathrm{m}$ (with respect to the nearby Ti-Fe blend at $2.189 \mu \mathrm{m}$ ) and (2) the slightly more marked "shoulder" just longward of the first CO band head around $2.295 \mu \mathrm{m}$. Still, considering the limited sampling in spectral types of the libraries we used and the possibility of veiling in the B component, we acknowledge that both components may well have the same spectral type and that both estimates could be systematically offset.

Inspection of the V773 Tau A spectra revealed that the profile of the strongest photospheric lines were asymmetric in both epochs, with a red "shoulder" in 2003 and a "blue" one in 2006. To illustrate this point we extracted the profiles of the four strongest isolated lines in our spectra $(\mathrm{Mg} 2.107 \mu \mathrm{m}, \mathrm{Al}$ $2.110 \mu \mathrm{m}$, Al $2.117 \mu \mathrm{m}$, and $\mathrm{Ti} 2.189 \mu \mathrm{m}$ - the line split of the $\mathrm{Ti} / \mathrm{Si} 2.179 \mu \mathrm{m}$ doublet is just large enough to prevent us from using this feature in the average profiles) and computed the average profile using the relative strength of the line as a weight. The resulting line profiles (Figure 6, left panel) indicate that we have resolved V773 Tau A as a double-lined spectroscopic binary in the near-infrared, despite the relatively large rotational broadening (B2007). The Ab component is detected offset from Aa at about $+60 \mathrm{~km} \mathrm{~s}^{-1}$ in 2003 and at about $-75 \mathrm{~km} \mathrm{~s}^{-1}$. These

\footnotetext{
11 http://www.gemini.edu/sciops/instruments/nearir-resources/ $? \mathrm{q}=$ node $/ 10167$
} 

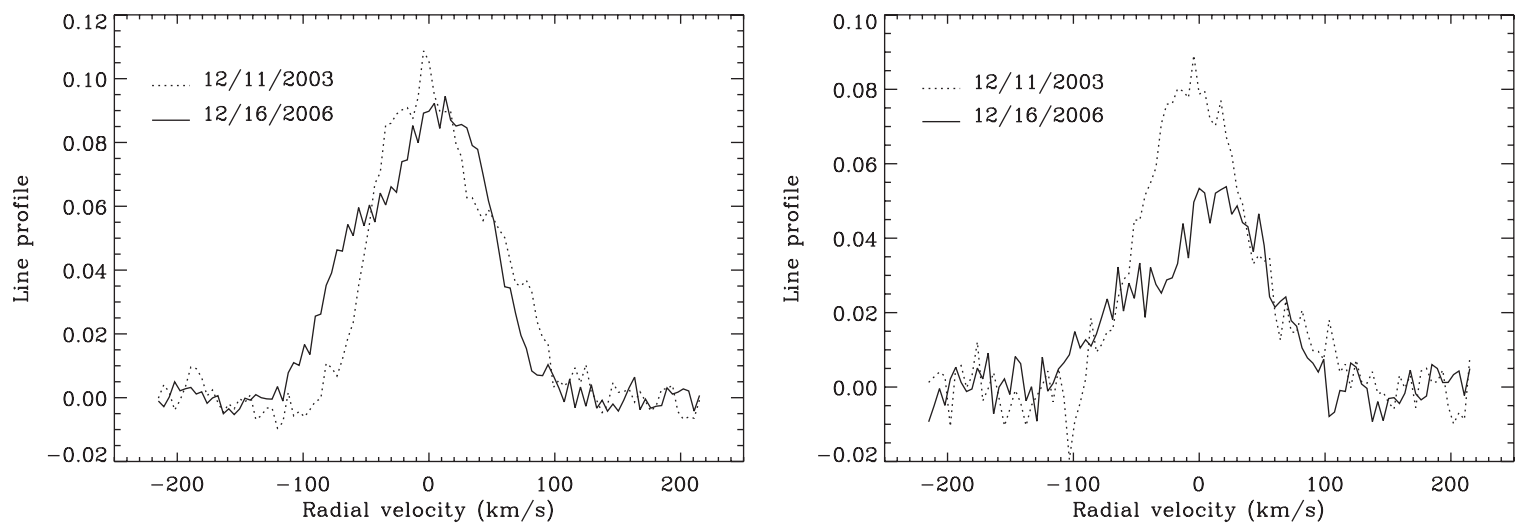

Figure 6. Average line profile for V773 Tau A (left) and V773 Tau B (right) based on the four most prominent isolated lines in our NIRSPEC spectra.

velocity offsets are in good agreement with the predictions from A-subsystem orbit modeling $\left(+54.4 \mathrm{~km} \mathrm{~s}^{-1}\right.$ and $-70.9 \mathrm{~km} \mathrm{~s}^{-1}$ in 2003 and 2006, respectively; B2007).

We further generated similar average line profiles for V773 Tau B in both epochs. We find the average line profile to be symmetric in 2003 but much broader and most likely double lined in 2006 (with a relative RV of about $-80 \mathrm{~km} \mathrm{~s}^{-1}$ for component $\mathrm{Bb}$ relative to $\mathrm{Ba}$ ). If confirmed in future observations of the system, this is the first direct evidence supporting the inference that V773 Tau B is itself an unresolved binary.

\section{DISCUSSION}

The most notable result from this analysis is the large value for the V773 Tau B dynamical mass, $2.35 \pm 0.67 M_{\odot}$. This $\mathrm{B}$-mass value is implied by the A-B orbit modeling described here and is independently supported by the companion VLBA study of T2012. The B dynamical mass estimate makes B comparable to, but slightly less massive than the A subsystem. Further, this B mass, along with the estimated luminosity (see below) and signs of double-lined features in our near-IR spectra (Section 3.3), suggests that like A, B is also a multiple stellar system. Before we discuss the implications of these results we need to consider the luminosity of V773 Tau B.

In modeling the V773 Tau B spectral energy distribution (SED), D2003 found a range of possible solutions as a function of possible line-of-sight extinction. In particular,

"With an additional 1 mag of extinction at $V$ toward V773 Tau [B], we find that its SED is well fitted by a K7 dwarf with a luminosity of $2.3 \pm 0.3 L_{\odot}$; a significant excess at $L^{\prime}$ remains present although the $H$ - and $K$-band fluxes are then consistent with photospheric levels."

Here we model the V773 Tau B SED informed by the mid-K spectral-type determination from Section 3.3. Resolved B photometry (White \& Ghez 2001; D2003) are well fitted by a range of mid-K SED templates $\left(T_{\text {eff }} \sim 4100-4300 \mathrm{~K}\right)$ from Pickles (1998), Lejeune et al. (1997), and PHEONIX (e.g., Allard et al. 2000), attenuated by considerable line-ofsight extinction (e.g., $A_{V} \sim 3 \mathrm{mag}$ ). To facilitate comparison with B2007 results on V773 Tau A, Figure 7 presents a model derived from the same template family (solar-abundance Kurucz-Lejeune template at $T_{\text {eff }}=4250 \mathrm{~K}$ and $\log g=$ 4.0). This model clearly fits the available photometry well $\left(\chi^{2} / \mathrm{DOF}=0.68\right)$ and results in a B-luminosity estimate of $2.6 \pm 0.5 L_{\odot}$ with $A_{V}$ of $2.9 \pm 0.3 \mathrm{mag}$. This extinction is

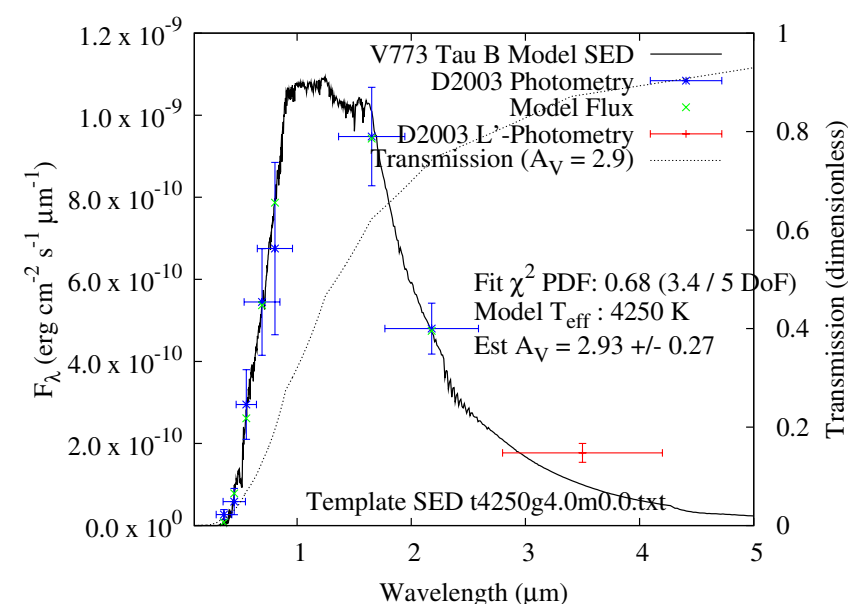

Figure 7. Sample V773 Tau B spectral energy distribution model. A sample SED model is shown using a $4250 \mathrm{~K}$ Kurucz-Lejunne SED template (Lejeune et al. $1997)$ with significant line-of-sight extinction $\left(A_{V}=2.9 \mathrm{mag}\right)$. The implied $\mathrm{B}$ luminosity is $2.6 \pm 0.5 L_{\odot}$. As first reported in D2003, the $L^{\prime}$ flux point (shown in red) indicates that there is a marginally significant excess compared with the estimated photospheric level.

roughly 1 mag higher in $A_{V}$ than reported toward A ( $A_{V}=1.8 \pm$ $0.2 \mathrm{mag}$ ) in B2007; taking the A-extinction value as interstellar this suggests a circum-B source for the $\sim 1$ mag of additional extinction found here. Like D2003, our SED modeling also finds a marginally significant $L^{\prime}$ excess, further reinforcing the hypothesis that there is circum- $\mathrm{B}$ material reprocessing radiation from B. However, the interpretation of this B SED modeling is complicated by the fact that $\mathrm{B}$ is seen to be variable (Figure 3), and the input data (White \& Ghez 2001; D2003) are not contemporaneous.

The combination of our B-component dynamical mass $\left(2.35 M_{\odot}\right)$ and luminosity estimate $\left(2.6 L_{\odot}\right)$ suggests a multiple system interpretation. For instance, D'Antona \& Mazzitelli (1997) models predict a luminosity of $17 L_{\odot}$ and $T_{\text {eff }} \sim 6200 \mathrm{~K}$ for a $3 \mathrm{Myr}$ (B2007) single star at $2.35 M_{\odot}$. (Note that a $1 \sigma$ excursion downward in the mass estimate- $1.7 M_{\odot}$-would predict $2.8 L_{\odot}$ and $5200 \mathrm{~K}$ from these same models.) Such a B luminosity and temperature would seem to be impossible based on the determinations presented here. However assuming the simplest multiple configuration for B as a binary arrangement with a pair of $1.175 M_{\odot}$ stars, the D'Antona \& Mazzitelli (1997) models predict a pair of such stars to have a total luminosity of $2.6 L_{\odot}$, and $T_{\text {eff }} \sim 4800 \mathrm{~K}$, in better agreement with the findings here. While mass arguments alone would not exclude a single star interpretation, combined with double-lined 
indications in the near-IR spectroscopic analysis (Section 3.3), the circumstantial case that V773 Tau B is a multiple stellar system seems strong. However, experience (e.g., T2002; D2003) has shown that one must consider the present $\mathrm{A}-\mathrm{B}$ orbit model as preliminary pending additional phase coverage, and the B-mass estimate is still significantly uncertain $(\sim 29 \% 1 \sigma)$.

Photometric variability in V773 Tau has typically been attributed to the B component (D2003; Figure 3) and interpreted as changing line-of-sight extinction from circum-B material. This interpretation is at least complicated both by potential B multiplicity and by the physical size of the A-B orbit (15.3 AU, Table 4). Dynamical studies (e.g., Artymowicz \& Lubow 1994; Pichardo et al. 2005) indicate that stable regions for diffuse material are offset from binaries by several times the orbital semimajor axis. Presumably, the outer radius of any stable circum-B orbit should be on the order of $15 / 3 \sim$ 5 AU. Similarly, the typical size of a putative B subsystem should be several times smaller than this 5 AU scale-probably $\leqslant 1$ AU-making the working model for B a short-period binary. In this picture, circumbinary material in the approximately 2-5 AU range would be responsible for both the near-infrared excess and photometric variability of $\mathrm{B}$. The large amount of dust needed to explain both extinction and variability (see below) raises the question whether it could remain dynamically stable in the region between $B$ as a putative short-period binary and the larger A-B orbit, and whether such a reservoir of material would show other observable signatures such as detectable thermal IR and millimeter flux. As a system V773 Tau shows a large mid-IR flux above photospheric levels (e.g., Prusti et al. 1992; D2003, Spitzer Taurus Legacy program; Padgett et al. 2011, http://irsa.ipac.caltech.edu/data/SPITZER/Taurus/), and Andrews \& Williams (2005) list significant flux at $850 \mu \mathrm{m}$ and $1.3 \mathrm{~mm}$. However, the presence of the V773 Tau C component (D2003; Woitas 2003) significantly complicates the interpretation of these flux measurements. A spatially resolved millimeter study with a sensitive facility such as ALMA would clarify the distribution of circumstellar material in the V773 Tau system.

If the putative $\mathrm{B}$ variability is due to changing line-ofsight extinction, this degree of variability is extraordinary. To account for the amplitude of the $K$ variability shown in Figure 3, a variation in $A_{V}$ of up to $25 \mathrm{mag}$ (assuming interstellar medium-like dust opacity) would be indicated. Such large $A_{V}$ excursions seem discrepant with the results of the SED modeling above. A more plausible alternative might be an optically thick structure-perhaps a disk that may or may not be perturbed by complicated dynamics-obscuring the line of sight to B components, allowing only a small amount of scattered light to reach the observer. Variability on a short timescale could be related to one of the components of B temporarily coming into direct view along its orbit, akin to the KH 15D system (Herbst et al. 2010, and references therein). The current sampling of the light curve for B is too sparse to decisively test this hypothesis. In either case, this large attenuation could explain why no clear detection of $\mathrm{B}$ is seen in extensive optical spectroscopic monitoring (Welty 1995; B2007, Table 2).

If the inference of B multiplicity and circum-B material is correct, then the V773 Tau system seems remarkably similar to the HD 98800 system, where circumbinary material is found around one but not both binaries in the system (Prato et al. 2001). In HD 98800 the inner B and outer A-B orbit significantly complicate the dynamics of B circumbinary material, leading to an inference of disk truncation and warping (Boden et al. 2005;
Akeson et al. 2007; Verrier \& Evans 2007; Pichardo et al. 2008). The apparent increasing system complexity raises questions of stability in V773 Tau. While more information on the B and C components will be necessary to address the specific stability of V773 Tau, it is clear that such complex systems are a common outcome of the star formation process and can remain stable for Gyr (e.g., Duquennoy \& Mayor 1991; Tokovinin 2008; Raghavan et al. 2010).

Some of the data presented here were obtained at the W.M. Keck Observatory, which is operated as a scientific partnership among the California Institute of Technology, the University of California, the University of Hawaii, and NASA. The Observatory was made possible by the generous financial support of the W.M. Keck Foundation. We gratefully acknowledge personnel from the W.M. Keck Observatory in supporting observations of V773 Tau. The authors recognize and acknowledge the very significant cultural role and reverence that the summit of Mauna Kea has always had within the indigenous Hawaiian community. We are most fortunate to have the opportunity to conduct observations from this mountain.

The authors gratefully acknowledge research support provided by the National Science Foundation, the California Institute of Technology, Harvard University, the University of California, DGAPA, UNAM, and CONACyT, Mexico. In particular: A.F.B. acknowledges support from NSF grant AST-0908822, G.T. acknowledges support from NSF grant AST-1007992, R.M.T. acknowledges support by the Deutsche Forschungsgemeinschaft (DFG) through the Emmy Noether Research grant VL 61/3-1. L.L. acknowledges the financial support of the Guggenheim Foundation and the von Humboldt Stiftung. Portions of this work were performed under the auspices of the U.S. Department of Energy by Lawrence Livermore National Laboratory under Contract DE-AC52-07NA27344.

\section{REFERENCES}

Akeson, R. L., Rice, W. K. M., Boden, A. F., et al. 2007, ApJ, 670, 1240 Allard, F., Hauschildt, P. H., \& Schweitzer, A. 2000, ApJ, 539, 366 Andrews, S., \& Williams, J. 2005, ApJ, 631, 1134

Artymowicz, P., \& Lubow, S. 1994, ApJ, 421, 651

Boden, A. F., Creech-Eakman, M. J., \& Queloz, D. 2000, ApJ, 536, 880 Boden, A. F., Sargent, A. I., Akeson, R. L., et al. 2005, ApJ, 635, 442 Boden, A. F., Torres, G., Sargent, A. I., et al. 2007, ApJ, 670, 1214 (B2007) Chauvin, G., Lagrange, A.-M., Bonavita, M., et al. 2010, A\&A, 509, 52 D’Antona, F., \& Mazzitelli, I. 1997, Mem. Soc. Astron. Ital., 68, 807 Duchêne, G., Ghez, A. M., McCabe, C., \& Weinberger, A. J. 2003, ApJ, 592, 288 (D2003)

Duquennoy, A., \& Mayor, M. 1991, A\&A, 248, 485

Figer, D. F., Gilmore, D., Kim, S. S., et al. 2003, ApJ, 599, 1193

Ghez, A. M., Neugebauer, G., \& Matthews, K. 1993, AJ, 106, 2005

Ghez, A. M., Salim, S., Weinberg, N. N., et al. 2008, ApJ, 689, 1044

Herbst, W., LeDuc, K., Hamilton, C. M., et al. 2010, AJ, 140, 2025

Ivanov, V. D., Rieke, M. J., Engelbracht, C. W., et al. 2004, ApJS, 151, 387

Kleinman, S., \& Hall, D. 1986, ApJS, 62, 501

Konopacky, Q. M., Ghez, A. M., Barman, T. S., et al. 2010, ApJ, 711, 1087

Leinert, Ch., Zinnecker, H., Weitzel, N., et al. 1993, A\&A, 278, 129

Lejeune, Th., Cuisinier, F., \& Buser, R. 1997, A\&AS, 125, 229

Lenzen, R., Hartung, M., Brandner, W., et al. 2003, Proc. SPIE, 944, 4841

Lestrade, J.-F., Preston, R. A., Jones, D. L., et al. 1999, A\&A, 344, 1014 (L1999)

Martín, E. L., Rebolo, R., Magazzu, A., \& Pavlenko, Ya. V. 1994, A\&A, 282, 503

Massi, M., Forbrich, J., Menten, K. M., et al. 2006, A\&A, 453, 959

Massi, M., Menten, M., \& Neidhofer, J. 2002, A\&A, 382, 152

Massi, M., Ros, E., Menten, K. M., et al. 2008, A\&A, 480, 489

McLean, I. S., Graham, J. R., Becklin, E. E., et al. 2000, Proc. SPIE, 4008, 1048 Padgett, D., et al. 2011, http://irsa.ipac.caltech.edu/data/SPITZER/Taurus/

Pichardo, B., Sparke, L. S., \& Aguilar, L. A. 2005, MNRAS, 359, 521

Pichardo, B., Sparke, L. S., \& Aguilar, L. A. 2008, MNRAS, 391, 815 
Pickles, A. 1998, PASP, 110, 863

Phillips, R. B., Lonsdale, C. J., Feigelson, E. D., \& Deeney, B. D. 1996, AJ, 111,918

Prato, L., Ghez, A. M., Pia, R. K., et al. 2001, ApJ, 549, 590

Prusti, T., Clark, F. O., Laureijs, R. J., Wakker, B. P., \& Wesselius, P. R. 1992, A\&A, 259, 537

Raghavan, D., McAlister, H. A., Henry, T. J., et al. 2010, ApJS, 190, 1

Rayner, J., Cushing, M., \& Vacca, W. 2009, ApJS, 185, 289

Rousset, G., Lacombe, F., Puget, P., et al. 2003, Proc. SPIE, 4839, 140

Rydgren, A., Strom, S., \& Strom, K. 1976, ApJS, 30, 307

Tamazian, V. S., Docobo, J. A., White, R. J., \& Woitas, J. 2002, ApJ, 578, 925 (T2002)
Tokovinin, A. 2008, MNRAS, 389, 925

Torres, G., Boden, A. F., Latham, D. W., Pan, M., \& Stefanik, R. P. 2002, AJ, 124,1716

Torres, G., Stefanik, R. P., Andersen, J., et al. 1997, AJ, 114, 2764

Torres, R., Loinard, L., Mioduszewski, A. J., et al. 2012, ApJ, 747, 18 (T2012)

Verrier, P., \& Evans, N. 2007, MNRAS, 390, 1377

Wallace, L., \& Hinkle, K. 1996, ApJS, 107, 312

Welty, A. 1995, AJ, 110, 776 (W1995)

White, R., \& Ghez, A. 2001, ApJ, 556, 265

Wizinowich, P. L., Acton, D. S., Lai, O., et al. 2000, Proc. SPIE, 4007, 2

Woitas, J. 2003, A\&A, 406, 685 